\title{
Alternative Criteria for Predictive Blind Deconvolution
}

\author{
Renan D. B. Brotto, Kenji Nose-Filho and João M. T. Romano
}

\begin{abstract}
Blind deconvolution is a major theme in signal processing and has been intensely investigated over the last decades. Among its several applications, we can mention the problem of seismic deconvolution and channel equalization in telecommunications. In these two cases, predictive techniques have been studied by different authors, and presented satisfactory results when some suitables conditions were fulfilled. In fact, the predictive deconvolution structure, when associated with the classical mean squared error criterion is only effective when the distortion system is minimum phase. In the case of nonminimum phase systems, it only provides magnitude equalization, but the phase response remains distorted. In order to overcome this problem, we present in this work some interesting results obtained with the use of $\ell_{p}$ norms, with $p \neq 2$, as optimization criteria.

First we demonstrate that the $\ell_{p}$ prediction error filter works as the Maximum Likelihood solution for blind deconvolution when the signal to be recovered has a generalized Gaussian distribution, with i.i.d. (identically and independently distributed) samples. From this, we show how the best $p$ can be chosen according to the signal distribution. Then we further investigate the phase response of the $\ell_{p}$ filter, emphasizing its potential as well as some limitations in dealing with blind deconvolution, even for nonminimum phase distortion system. Finally, some performance simulations results are provided.
\end{abstract}

Index Terms-Predictive deconvolution, $\ell_{p}$ norms, nonminimum phase deconvolution.

\section{INTRODUCTION}

The problem of estimating future values of a time series from its present and past samples is a very challenging problem in the field of signal processing. Its origins goes back to the works of Kolmogorov [1], in the context of stationary discrete time signals, and Wiener [2], where the continuous time optimal predictor is formulated.

The objective in prediction consists in finding a mapping $F[\cdot]$ that, when applied to a set of past samples of a time series $\mathbf{x}(n-1)=[x(n-1) x(n-2) \cdots x(n-L)]^{T}$, results in an estimative to its present value $x(n)[3],[4]$ :

The authors acknowledge CAPES and FAPESP (2017/13025-2) for financial support

Renan D. B. Brotto and João M. T. Romano are with the School of Electrical and Computer Engineering, State University of Campinas (UNICAMP), campus Campinas, São Paulo, Brazil (e-mail: \{rbrotto, romano\}@decom.fee.unicamp.br)

Kenji Nose-Filho is with Center of Engineering, Modeling and Applied Social Sciences, Federal University of ABC (UFABC), campus Santo André, São Paulo, Brazil (e-mail: kenji.nose @ufabc.edu.br)

Digital Object Identifier: 10.14209/jcis.2018.27

$$
F[\mathbf{x}(n-1)]=\hat{x}(n) .
$$

The generic mapping in (1) may be associated to a particular filter structure, with a set of parameters to be adjusted. For the case of linear prediction, this mapping can be represented by a finite impulse response (FIR) filter, namely prediction filter, which input-output relation is given by:

$$
\hat{x}(n)=\sum_{i=1}^{L} w_{i} x(n-i)=\mathbf{w}^{T} \mathbf{x}(n-1),
$$

where $w_{1}, w_{2}, \cdots, w_{L}$ are the prediction coefficients, $x(n-i)$, for $i=1,2, \ldots L$, are the past samples of $x(n)$, and $\hat{x}(n)$ is its estimative. Moreover, we can define $\mathbf{w}=\left[\begin{array}{llll}w_{1} & w_{2} & \cdots & w_{L}\end{array}\right]^{T}$ as the vector of parameters to be adjusted. The difference between $x(n)$ and $\hat{x}(n)$ leads to the so called prediction error [5]:

$$
e(n)=x(n)-\hat{x}(n)=x(n)-\sum_{i=1}^{L} w_{i} x(n-i) .
$$

Meanwhile (2) stands for the prediction filter, the inputoutput relation given by (3) stands for what we call the prediction error filter (PEF), which structure is illustrated by the diagram of Figure 1.

Prediction Error Filter

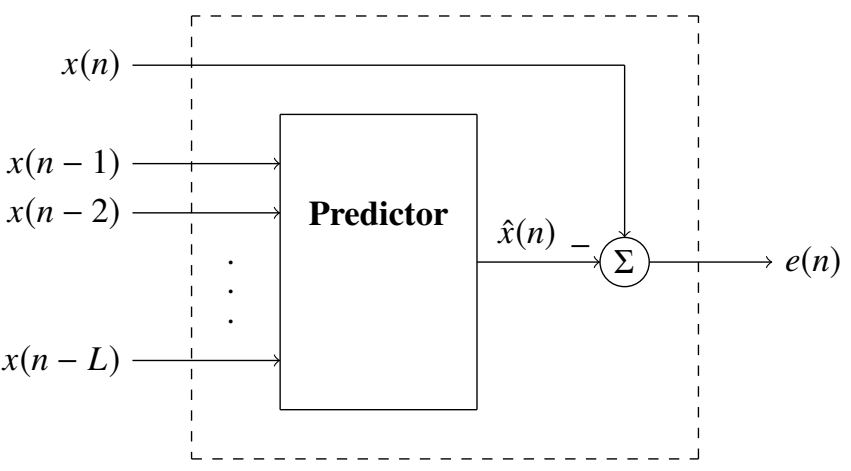

Fig. 1: The prediction filter consists in mapping the past samples of $x(n)$ in its estimative $\hat{x}(n)$. The prediction error filter stands for the mapping of $x(n)$ and its past samples in the prediction error $e(n)$.

The problem of linear prediction, as described above, is of great relevance for the field of signal processing, and can be applied for both parametric and nonparametric models. It can be used to estimate the parameters of autoregressive (AR) 
models [6], such as in the linear predictive coding of speech signals; in time series forecasting [7]; and in the deconvolution problem, which is the problem of interest in this work [3].

It is well known in the literature that the classical prediction error filter obtained through the mean squared error (MSE) criterion is only effective for the deconvolution of minimum phase systems, since this filter provides decorrelated output samples. Hence, for signals that have been distorted by nonminimum phase systems, the classical PEF equalizes only magnitude, but the phase response remains distorted.

To overcome this problem, we need to explore a stronger hypothesis: the independence between the samples. This can be attained by means of a nonlinear PEF structure adjusted by the MSE criterion, as proposed in [8], [9], or rather, maintaining the linear PEF structure but employing alternative criteria. We have investigated the latter solution in [10] and presented some preliminary results. This work introduced an approach based on $\ell_{p}$ PEF, showing that it works as a Maximum Likelihood solution for blind deconvolution when the signal to be recovered has a generalized Gaussian distribution.

The present paper extends the study in [10], investigating the zero location and phase response of the $\ell_{p}$ PEF, and presents several new results. Such results concern specially a deeper assessment about the zero location (and then, the phase response) of the $\ell_{p} \mathrm{PEF}$, when the value of $p$ is adjusted according to the properties of sparsity and antisparsity [11] of the signal to be recovered. All the results have been obtained in a noise free scenario with real valued signals.

The paper is organized as follows: In Section II we revisit the principle and main properties of the classical MSE PEF. Then, in Section III, we introduce the problem of predictive deconvolution, emphasizing the limits of the MSE solution. In Section IV we demonstrate that the $\ell_{p}$ PEF works as the Maximum Likelihood solution for blind deconvolution when the signal to be recovered has a generalized Gaussian distribution; from this, we show how the best $p$ can be chosen in according to the signal distribution. Then, in Section V, we provide a deeper study about the phase response of the $\ell_{p}$ filter, emphasizing its potential as well as some limitations in dealing with blind deconvolution, even for nonminimum phase distortion system. In Section VI we evaluate the performance of the $\ell_{1}$ deconvolution for a sparse signal and the $\ell_{\infty}$ deconvolution for an antisparse signal, considering first and second order channels, with minimum and maximum phase responses. Finally, in Section VII we close the paper with some conclusion remarks and perspectives of future works.

\section{THE MEAN SQUARED PREDICTION ERROR FILTER REVISITED}

The prediction coefficients $w_{i}, i=1, \ldots, L$ are adjusted in order to minimize a given cost function $J(\mathbf{w})$, which, classically, is chosen as the mean squared error criterion:

$$
\begin{aligned}
J(\mathbf{w}) & =\mathbb{E}\left[e^{2}(n)\right]=\mathbb{E}\left[\left(x(n)-\mathbf{w}^{T} \mathbf{x}(n-1)\right)^{2}\right]= \\
& =\mathbb{E}[x(n) x(n)]-\mathbb{E}\left[x(n) \mathbf{x}^{T}(n-1)\right] \mathbf{w}+ \\
& -\mathbf{w}^{T} \mathbb{E}[\mathbf{x}(n-1) x(n)]+ \\
& +\mathbf{w}^{\mathbf{T}} \mathbb{E}\left[\mathbf{x}(n-1) \mathbf{x}^{T}(n-1)\right] \mathbf{w},
\end{aligned}
$$

where $\mathbb{E}[\cdot]$ stands for the expectation operator.

Besides the signal variance $\sigma_{x}^{2}=\mathbb{E}[x(n) x(n)]$, other two terms completely characterize $J(\mathbf{w})$ : the autocorrelation matrix $\mathbf{R}=\mathbb{E}\left[\mathbf{x}(n-1) \mathbf{x}^{T}(n-1)\right]$, and the autocorrelation vector $\mathbf{p}=$ $\mathbb{E}[\mathbf{x}(n-1) x(n)]$. Rewriting (4) in terms of $\sigma_{x}^{2}, \mathbf{R}$, and $\mathbf{p}$ results in:

$$
J(\mathbf{w})=\sigma_{x}^{2}-\mathbf{p}^{T} \mathbf{w}-\mathbf{w}^{T} \mathbf{p}+\mathbf{w}^{T} \mathbf{R} \mathbf{w} .
$$

The cost function in (5) is a quadratic function of the adjustable parameters and describes an elliptic paraboloid with one single minimum point. To determine this point, we set the gradient equal to the null vector:

$$
\nabla J(\mathbf{w})=2 \mathbf{R w}-2 \mathbf{p}=\mathbf{0} \rightarrow \mathbf{R w}=\mathbf{p} .
$$

The solution of (6) is known in the literature as Wiener's solution [3] and is given by:

$$
\mathbf{w}^{*}=\mathbf{R}^{-1} \mathbf{p} .
$$

Now, in order to introduce the relationship between linear prediction and deconvolution, let us consider two properties of the mean squared prediction error filter, given by (7).

Due to the equivalence between MSE minimization and the orthogonalization procedure, the error produced by the Wiener filter is uncorrelated with its input [3]:

$$
\mathbb{E}[e(n) x(n-i)]=0,1 \leq i \leq L .
$$

Since the prediction error $e(n)$ is a linear combination of the past samples of $x(n)$, we have, from (3) and (8):

$$
\mathbb{E}[e(n) e(n-k)]=0, \quad \forall k>1,
$$

which means that, for a sufficient number of coefficients $L$, the PEF tends to generate an uncorrelated (white) output error.

Another property of the prediction error filter is related to its phase response: in fact, it is possible to show that all of its zeros lay inside the unit circle, so that the PEF is a minimum phase filter [5].

\section{PREdictive DeCONVOLUTION AND BLind EQUALIZATION OF MINIMUM PHASE SYSTEMS}

These two properties of the mean squared prediction error filter lead us to an interesting result: the possibility of removing, or attenuating, the effects of linear and time invariant systems. This problem is known as the predictive deconvolution [3]. The first application of predictive deconvolution goes back to Robinson's work [12] in the context of seismic deconvolution.

In seismic data acquisition, an artificially and controlled wave is emitted and, due to the difference between the acoustic 
impedance of the different earth layers, part of its energy is reflected back to the surface. The reflected signals are captured by sensors, originating the so called seismic traces. Therefore, the seismic trace corresponds to the convolution between the seismic wavelet and the earth impulse response.

From convolution commutative property [13] we can consider the earth impulse response as an input signal $s(n)$ and the seismic wavelet as a system impulse response $h(n)$, as shown in Figure 2:

\begin{tabular}{|c|c|c|}
\hline Earth Impulse Response & Seismic wavelet & Seismic Trace \\
\hline$s(n)$ & $h(n)$ & $x(n)$ \\
\hline
\end{tabular}

Fig. 2: Simple model of the seismic data acquisition. The seismic trace $x(n)$ can be seen as the convolution of the seismic wavelet impulse response $h(n)$ with the earth impulse response $s(n)$.

\begin{tabular}{c|c|c} 
Seismic Trace & $\begin{array}{c}\text { Prediction Error Filter } \\
w(n)\end{array}$ & $\begin{array}{c}\text { Earth Impulse Response } \\
\text { Estimative }\end{array}$ \\
& $\hat{s}(n)$
\end{tabular}

Fig. 3: Simple model of seismic deconvolution. The prediction error filter is applied in order to remove the effects introduced by the seismic wavelet, providing an estimative of the earth impulse response.

So, the output seismic trace is given by:

$$
x(n)=s(n) * h(n) .
$$

Robinson's approach consists in applying a prediction error filter to the signal $x(n)$ in order to remove, or attenuate, the effects introduced by the seismic wavelet. Thus, the recovery of the earth impulse response is based in two hypotheses [12]:

1) The earth impulse response behaves like a white noise (i.e. all of its samples are uncorrelated);

2) The seismic wavelet corresponds to the impulse response of a minimum phase system.

Under these two simplifying hypothesis, it is possible to show that a sufficient length prediction error filter is able to remove the effects introduced by the seismic wavelet, as depicted in Figure 3.

Another related problem of interest is that of Channel Equalization, in which the main goal is to retrieve the signal transmitted though a communication channel, usually modeled by an FIR filter. The received signal is given by the convolution of the transmitted signal with the impulse response of the channel. In this case, a prediction error filter can be used to perform deconvolution if the transmitted signal is composed of a sequence of uncorrelated samples and the channel is minimum phase.

An important difference between the above mentioned problems, seismic deconvolution and channel equalization, is related to the characteristics of the input signals. In seismic deconvolution, the samples of the earth impulse response can be modeled by i.i.d. sparse and continuous random variables [14]. On the other hand, the samples of the transmitted signal in the channel equalization problem are usually modeled by i.i.d. discrete and uniformly distributed random variable [15]. However, in both cases, the seismic wavelet and the communication channel may be given by a nonminimum phase system [16], [17].

In the case of nonminimum phase systems, the mean squared prediction error filter is not able to remove nor attenuate the effects of the seismic wavelet or the communication channel. It provides only amplitude equalization, i.e., it acts as a whitening filter only. In other words, to explore the decorrelation property of the signal to be recovered is not sufficient to provide blind deconvolution when the distortion system is nonminimum phase. To overcome this limitation, we aim to search for a cost function that could explore a stronger property of the desired signal $s(n)$ : the i.i.d. hypothesis. In fact, while correlation provides only second order statistic information, exploring independence implies dealing with all the statistic behavior of the signal, so that it could be properly recovered, as explained next.

\section{THE $\ell_{p}$ PEF AS AN ALTERNATIVE FOR BLIND DECONVOLUTION}

As posed in the previous sections, the MSE criterion is given by the equation (4) and deals with the second order statistic behavior of the signals. Once such information is not always available in practical scenarios, an alternative consists in using temporal means of the prediction error signal, as follows:

$$
J_{L S}(\mathbf{w})=\sum_{n=0}^{N-1} \lambda(n) e^{2}(n),
$$

where $\lambda(n)$ is a weight factor that controls the degree of relevance of the error sample at time instant $n, e(n)$ is the error signal and $\mathbf{w}$ are the filter coefficients.

Considering stationary and ergodic processes [18], the criteria given by (4) and (11) are equivalent for practical purposes. So, minimizing the MSE criterion is equivalent to minimize the least squares (LS) criterion in (11).

A particular version of (11) is obtained when we use $\lambda(n)=$ $\frac{1}{N}$, for all $n$ :

$$
J_{L S}(\mathbf{w})=\frac{1}{N} \sum_{n=0}^{N-1} e^{2}(n) .
$$

From an optimization point of view, the scaling factor $1 / N$ does not change the value for the optimal filter $\mathbf{w}^{*}$ and so we have an equivalence between the two optimization problems:

$$
\underset{\mathbf{w}}{\arg \min } \frac{1}{N} \sum_{n=0}^{N-1} e^{2}(n)=\underset{\mathbf{w}}{\arg \min } \sum_{n=0}^{N-1} e^{2}(n) .
$$

Considering the definition of $\ell_{p}$ norm [19]

$$
\|e\|_{p}=\left(\sum_{n=0}^{N-1}|e(n)|^{p}\right)^{1 / p}
$$


we have that the right hand side in equation (13) corresponds to the square of $\ell_{2}$ norm of the prediction error. From the previous analysis, we have that, for our purposes here, the MSE criterion is equivalent to the $\ell_{2}$ norm.

As said in the previous section, the $\ell_{2}$ norm (or MSE criterion) can only provide linear decorrelation, what can be seen as a weak independence, and is efficient when dealing only with minimum phase systems. From this we can verify two restrictions of the $\ell_{2}$ norm: one with respect to a signal property (linear decorrelation), and the second as a system restriction (minimum phase).

Our objective is to investigate how $\ell_{p}$ norms, with $p \neq 2$, can deal with these restrictions. For this, let us analyze these norms from two different perspectives:

1) $\ell_{p}$ norm as a Maximum Likelihood estimator for generalized Gaussian distribution, in order to take advantage of the signal probability distribution as well as sparsity properties;

2) $\ell_{p}$ PEF, with $p \neq 2$, as a nonminimum phase system, showing the potentiality to deal with the minimum phase restriction.

In the next subsections we show the development of these two perspectives of the $\ell_{p}$ prediction error filters, as summarized in Figure 4:

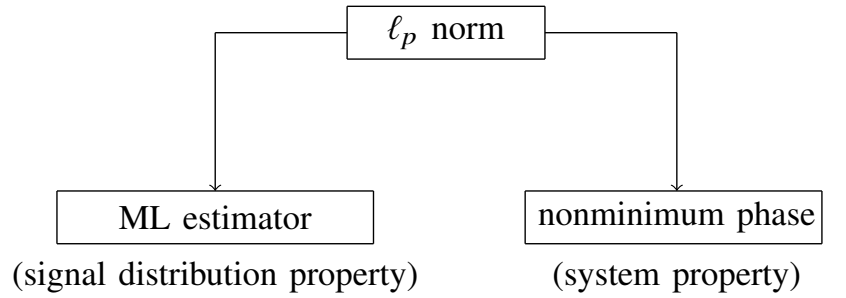

Fig. 4: Summary of the two perspectives of $\ell_{p}$ PEF: Maximum Likelihood estimators and nonminimum phase response.

\section{A. $\ell_{p}$ norms and Maximum Likelihood Criterion}

The Maximum Likelihood (ML) criterion can be viewed as a particular case of the Infomax principle [20]. It was derived in the context of neural networks and used in the Blind Source Separation problem [21], [3], which can be seen as an extension of the deconvolution problem. To obtain the ML criterion, we just need to set the nonlinearities $f_{i}(\cdot)$ as the cumulative distribution functions of the sources [3]. Cardoso's work (1998) [22] presents a deep study (in the context of BSS) of this criterion, given by:

$$
\max _{\mathbf{W}} J_{\text {Infomax }}(\mathbf{W})=\mathbb{E}\left[\sum_{i=1}^{M} \log \left(p_{s_{i}}\left(\mathbf{w}_{\mathbf{i}} \mathbf{x}\right)\right)\right]+\log (|\operatorname{det}(\mathbf{W})|),
$$

where $p_{s_{i}}(\cdot)$ is the probability distribution function of the $i$-th source, for $i=1,2, \cdots M$.

By applying this criterion into the deconvolution task, the following optimization problem holds:

$$
\max _{\mathbf{W}} J_{M L}=\max _{\mathbf{W}} \mathbb{E}_{N}\left[\sum_{i=1}^{N} \log \left(p_{s}\left(\mathbf{w}_{\mathbf{i}} \mathbf{x}\right)\right)\right],
$$

where $\mathbb{E}_{N}$ represents the temporal mean of the $N$ available data; $\mathbf{W}$ is the convolution matrix associated with the filter $w(n) ; \mathbf{w}_{\mathbf{i}}$ is its $i$-th row; $p_{S}(\cdot)$ is the probability distribution of the input signal $s(n)$; and $\mathbf{x}=[x(0) x(1) \cdots x(N-1)]^{T}$.

The Maximum Likelihood criterion provides an unbiased and efficient estimator, i.e., its variance reaches the CrammerRao limit as the number of samples goes to infinity [3], [23]. However, it requires the explicit knowledge of the input signal distribution function. Fortunately, many distributions with real applications can be unified in the generalized Gaussian distribution [24], given by:

$$
p_{S}(s)=\frac{\beta}{2 \alpha \Gamma(1 / \beta)} e^{-\left(\frac{|s-\mu|}{\alpha}\right)^{\beta}},
$$

where $\alpha$ is the dispersion parameter and $\beta$ is the shape parameter, both related by:

$$
\alpha^{\beta}=\beta \mathbb{E}\left[|s|^{\beta}\right] .
$$

For $\beta=1$, (17) corresponds to the Laplacian distribution; if $\beta=2$, we have the classical Gaussian distribution; and as $\beta \rightarrow \infty$, we obtain the uniform distribution.

Applying the Maximum Likelihood criterion (using the natural base for the logarithm) in the generalized Gaussian distribution, with $\mu=0$, we have:

$$
\begin{aligned}
J_{M L}(\mathbf{W}) & =\mathbb{E}_{N}\left[\sum_{i=1}^{N} \ln \left(p_{s}\left(\mathbf{w}_{\mathbf{i}} \mathbf{x}\right)\right)\right]= \\
& =\mathbb{E}_{N}\left[\sum_{i=1}^{N} \ln \left(\frac{\beta}{2 \alpha \Gamma(1 / \beta)}\right)-\frac{\left|\mathbf{w}_{\mathbf{i}} \mathbf{x}\right|^{\beta}}{\alpha^{\beta}}\right]= \\
& =N \ln \left(\frac{\beta}{2 \alpha \Gamma(1 / \beta)}\right)-\frac{1}{\alpha^{\beta}} \mathbb{E}_{N}\left[\sum_{i=1}^{N}\left|\mathbf{w}_{\mathbf{i}} \mathbf{x}\right|^{\beta}\right]= \\
& =N \ln \left(\frac{\beta}{2 \alpha \Gamma(1 / \beta)}\right)-\frac{1}{\alpha^{\beta}} \mathbb{E}_{N}\left[\|e\|_{\beta}^{\beta}\right] .
\end{aligned}
$$

Once the parameters $\alpha$ and $\beta$ are fixed, we have the following optimization problem:

$$
\max _{\mathbf{W}} J_{M L}(\mathbf{W})=\max _{\mathbf{W}}-\mathbb{E}_{N}\left[\|e\|_{\beta}^{\beta}\right]=\min _{\mathbf{W}} \mathbb{E}_{N}\left[\|e\|_{\beta}^{\beta}\right] .
$$

Equation (20) shows that maximizing the Likelihood criterion is equivalent to minimize the temporal mean of the associated $\ell_{p}$ norm. Also, this equation establishes a relation between the signal distribution and the more suitable value for $p$, as summarized next:

- For super Gaussian distributions [21], we choose $1 \leq p<$ 2 , with $p=1$ for Laplacian distribution.

- For a Gaussian distribution, we choose $p=2$;

- For sub Gaussian distributions [21], we adopt $p>2$, with $p \rightarrow \infty$ for uniform distribution.

From these results we observe two dual potential scenarios for predictive deconvolution:

1) the case where the desired signal is super Gaussian (sparsity property to be explored) and an $\ell_{p}$ predictor may be used with $1 \leq p \leq 2$ and 
2) the case where the desired signal is sub Gaussian (antisparsity property [11] to be explored) and it is suitable to use $p>2$.

\section{B. $\ell_{p}$ norms and the PEF phase response}

The location of the PEF zeros has been investigated in a number of works, especially during the 70s and 80s [25][27]. Usually, in the context of application of these works, the classical MSE (or $\ell_{2}$ ) criterion was employed.

In order to get phase information in the deconvolution problem, alternative approaches have explored the use of $\ell_{p}$ norms, with $p \neq 2$, as a criterion to optimize the PEF coefficients. To the best of our knowledge, the work by Scargle [28] derived the first results on the location of $\ell_{p}$ PEF's zeros, for $p \neq 2$. The work of Bednar [29] showed an application in seismic predictive deconvolution. The work of Knockaert [30] showed that all $\ell_{p}$ predictors (with $p \neq 2$ ) are unstable, in the sense that some of its zeros are outside the unit circle. Knockaert's paper also claimed that such zeros cannot go outside of a circle with radius 2. Hence, Knockaert's works shows the potentiality of $\ell_{p}$ (with $p \neq 2$ ) PEF, since the predictor can put some zeros outside the unit circle, but also shows a restriction: the filter zeros cannot be located in an arbitrary point of the complex plane.

These instigating results motivate us to perform a deeper study about the $\ell_{p}$ PEF zeros location, analyzing how flexible the filter phase response can be and how much it impacts the deconvolution performance. Next, we show the results obtained from this study in our context of application.

\section{ASSESSMENT On THE BeHAVIOR OF THE $\ell_{p}$ PEF PHASE RESPONSE}

In order to study the $\ell_{p}$ PEF phase response, this section has a twofold objective.

First, we obtain a set of simulations results. Then, we assess more deeply the behavior of the PEF zeros by approximating a situation in which we have a one pole distortion system. The proposed FIR approximation allows us to study the location of the zeros of the $\ell_{1}$ and $\ell_{\infty}$ PEFs, according to this pole position.

\section{A. Duality between $\ell_{1}$ and $\ell_{\infty}$ filters}

With the optimization criteria chosen as the most suitable for each case, our objective here is to study the location of the $\ell_{1}$ and $\ell_{\infty}$ PEF zeros. For comparison, we have also considered the supervised case. In this situation, we perform $\ell_{1}$ and $\ell_{\infty}$ minimization of the error signal, which is produced by having access to the signal we are aiming to recover, possibly with a delay:

$$
e(n)_{\text {supervised }}=s(n-d)-y(n),
$$

with $y(n)=w(n) * x(n)$ as the filter output signal and $d$ as the delay in the reference signal.

The sparse signal is generated accordingly to a BernoulliGaussian distribution: we first generate a Bernoulli sequence with $\operatorname{Pr}\{s(n)=1\}=0.02$; then, we multiply each element of this sequence by a random variable $N(0,1)$ (note that we have a different realization of this variable for each sample of the sequence). For the uniform signal, we have chosen a binary alphabet $(+1,-1)$.

The experiments consider three typical cases of channel:

- Minimum phase: $H_{1}(z)=1+0.5 z^{-1}+0.3 z^{-2}$;

- Mixed phase: $H_{2}(z)=0.5+1 z^{-1}+0.3 z^{-2}$;

- Maximum phase: $H_{3}(z)=1+1.5 z^{-1}+1.8 z^{-2}$.

For the unsupervised case, we used a PEF with one zero (and so, just one adjustable coefficient, $p_{1}$ ), i.e., $P(z)=$ $1-p_{1} z^{-1}$. In the supervised scenario, we have also employed a deconvolution filter with one zero (but two adjustable coefficients, $w_{0}$ and $\left.w_{1}\right): W(z)=w_{0}+w_{1} z^{-1}$. After the optimization, we have normalized the filter in $w_{0}$, just for comparison with the corresponding PEF.

A very important issue for the supervised case is the optimal delay in the reference signal: zero delay for the minimum phase, one sample for the mixed phase and three samples for the maximum phase channel.

To minimize the $\ell_{1}$ norm, the filter coefficients were adjusted by the error signal LMS [31], while the $\ell_{\infty}$ PEFs coefficients were minimized with the populational meta heuristic of differential evolution [32], described in the Appendix of this paper.

The obtained results are depicted in Table I, where $P_{1}(z)$ and $P_{\infty}(z)$ denote the PEFs obtained by minimizing the $\ell_{1}$ and $\ell_{\infty}$ norms, respectively, and $W_{1}(z)$ and $W_{\infty}(z)$ denote the filters obtained by minimizing the error $\ell_{1}$ and $\ell_{\infty}$ norms, respectively, in the supervised case:

TABLE I: Deconvolution filters obtained in sparse and antisparse deconvolution for both supervised and unsupervised scenarios.

\begin{tabular}{cc}
\hline Supervised & Unsupervised \\
\hline \multicolumn{2}{c}{ Minimum phase channel } \\
$W_{1}(z)=1-0.4997 z^{-1}$ & $P_{1}(z)=1-0.5 z^{-1}$ \\
$W_{\infty}(z)=1-0.5000 z^{-1}$ & $P_{\infty}(z)=1-0.5 z^{-1}$ \\
\hline \multicolumn{3}{c}{ Mixed phase channel } \\
$W_{1}(z)=1-0.2993 z^{-1}$ & $P_{1}(z)=1-0.3 z^{-1}$ \\
$W_{\infty}(z)=1-0.2997 z^{-1}$ & $P_{\infty}(z)=1-0.3 z^{-1}$ \\
\hline \multicolumn{3}{c}{ Maximum phase channel } \\
$W_{1}(z)=1-1.4969 z^{-1}$ & $P_{1}(z)=1-1.2 z^{-1}$ \\
$W_{\infty}(z)=1-1.4885 z^{-1}$ & $P_{\infty}(z)=1-1.2 z^{-1}$ \\
\hline
\end{tabular}

From the Table I we have two interesting results:

- Minimizing the $\ell_{1}$ norm to retrieve a sparse signal leads to the same filters obtained by minimizing the $\ell_{\infty}$ norm for deconvolution of an antisparse signal, for all channels considered, in both supervised and unsupervised scenarios. This result confirms the duality relationships involving the $\ell_{p}$ norms and the signal distributions.

- Comparing the supervised and unsupervised filters, adjusted by both $\ell_{1}$ and $\ell_{\infty}$ norms, we have the same zeros for the minimum (zero at 0.5 ) and mixed (zero at 0.3) phase channels. On the other hand, for maximum phase response, the filters obtained are slightly different: the supervised one put its zero at 1.5 (for both criteria), while the PEF put its zero at 1.2 (for $\ell_{1}$ and $\ell_{\infty}$ ). From this difference we can see a limitation of the $\ell_{p}$ PEF, with 
$p=1$ or $p=\infty$, but also, we can see the potential of such a structure, since it positioned a zero outside the unit circle. This potential is deeply explored in the following.

\section{B. $\ell_{p}$ prediction error filter zeros}

In order to proceed with our study, let us first consider a very simple case of distortion system that can be easily deconvolved by an MSE PEF: a first order AR channel

$$
H(z)=\frac{1}{1-a z^{-1}}
$$

Deconvolution is clearly attained by applying a single zero $\ell_{p} \mathrm{PEF}$ :

$$
W(z)=1-w_{1} z^{-1}
$$

Clearly, $W(z)$ will put its zero on the same position of the channel pole, i.e., $w_{1}=a$, to eliminate all system distortion.

It is worth observing that the channel in (22) can be arbitrarily approximated by an FIR filter as follows:

$$
\frac{1}{1-a z^{-1}} \approx 1+a z^{-1}+a^{2} z^{-2}+\ldots+a^{K} z^{-K}=\sum_{i=0}^{K}\left(a z^{-1}\right)^{i}
$$

This kind of approximation is particularly suitable in our study since it allows to simulate the effect of a pole outside the unit circle, i.e., the case where $|a|>1$ in (22).

We can rewrite equation (24) in a more compact form:

$$
H(z)=\sum_{i=0}^{K}\left(a z^{-1}\right)^{i}=\frac{1-\left(a z^{-1}\right)^{K+1}}{1-a z^{-1}}
$$

Equation (25) shows that the channel zeros correspond to the $K+1$ complex roots [33], except the one at $a$. Therefore, the channel tries to approximate a pole by a crown of complex and symmetric zeros of magnitude $a$, except at $z=a$. When the complete inversion is achieved, the zeros of the combined response $H(z) W(z)$ (channel and filter) in the complex plane will form a complete crown with radius $a$.

To illustrate it, let us consider a channel of order $K=15$ (for symmetry purposes), adopting the $\ell_{2}$ norm as optimization criterion and adjusting the filter coefficients by differential evolution ${ }^{1}$, with a population of 30 individuals in 800 generations. In this first case, we have used $a=0.7$ (so the channel is minimum phase) and we have adopted an input signal with a Gaussian distribution $N(0,1)$. The result of this example is shown if Figure 5:

\footnotetext{
${ }^{1}$ When we use $\ell_{2}$ norm, we have a convex optimization problem, with a closed solution and so it is not necessary the use of a heuristic. But the differential evolution has shown a faster convergence when compared to the LMS algorithm, which, alongside the simplicity of this meta heuristic, justifies its application to this problem.
}

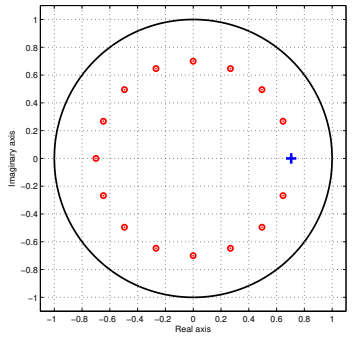

Fig. 5: Diagram of the channel (red circles) and filter (blue cross) zeros, for a Gaussian signal and $a=0.7$. We can see a crown of zeros, which indicates the complete inversion of the channel.

In Figure 5 we can see the crown of zeros, which indicates that the obtained filter completely inverts the channel, providing magnitude and phase equalization.

Repeating the experiment for $a=1.2$ (maximum phase channel) we have the following result:

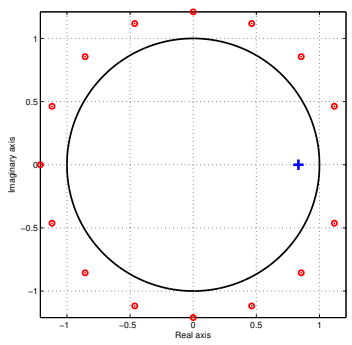

Fig. 6: Diagram of the channel (red circles) and filter (blue cross) zeros, for a Gaussian signal and $a=1.2$. In this case, the filter cannot put its zero outside the unit circle, elucidating the minimum phase restriction.

As we can see in Figure 6, the filter cannot put its zero outside the unit circle. Therefore, deconvolution is not achieved in this case: the filter performed only a decorrelation between the signal samples, but not independence. This is equivalent to provide magnitude equalization only.

Next, we will repeat the same experiment for $\ell_{1}$ and $\ell_{\infty}$ filters, again with a $K=15$ and a first order filter $W(z)=$ $1-w_{1} z^{-1}$.

For the input signal distribution we consider 3 cases:

1) Gaussian distribution, $N(0,1)$;

2) Bernoulli-Gaussian, $\operatorname{Pr}(s(n)=1)=0.02$ e $N(0,1)$;

3) Uniform distribution, with support $[-1,1]$.

In all cases, the filter coefficients were adjusted by differential evolution, with a population of 40 individuals in 2000 generations. We have also considered 2000 samples for the involved signals. The results for the $\ell_{1}$ filter are depicted in Figures 7, 8 and 9: 

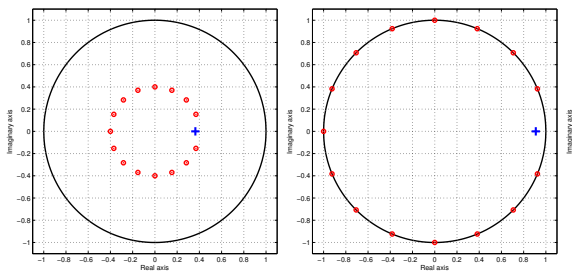

(a) $a=0.4$.

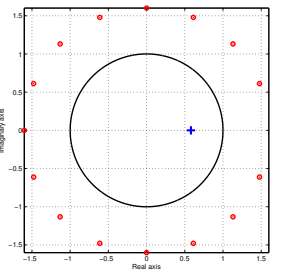

(c) $a=1.6$.

Fig. 7: Zero diagram for a $\ell_{1}$ filter and a Gaussian signal. For this type of signal, the $\ell_{1}$ PEF zero remained inside the unit circle.

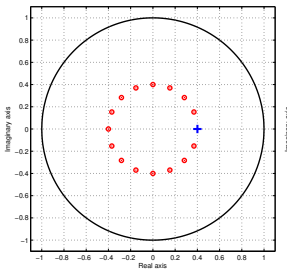

(a) $a=0.4$.

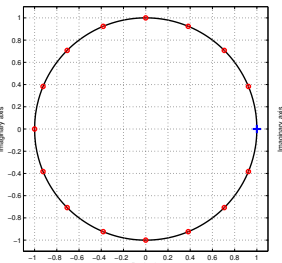

(b) $a=1$.

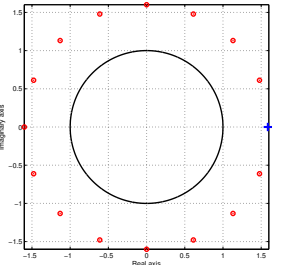

(c) $a=1.6$.

Fig. 8: Zero diagram for a $\ell_{1}$ filter and a Bernoulli-Gaussian signal. For this type of signal (i.e. a sparse one), the filter put its zero outside the unit circle, showing its potential to perform nonminimum phase deconvolution.

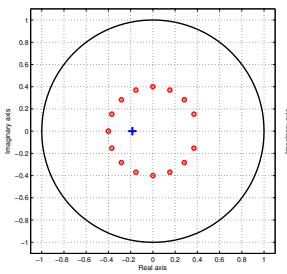

(a) $a=0.4$.

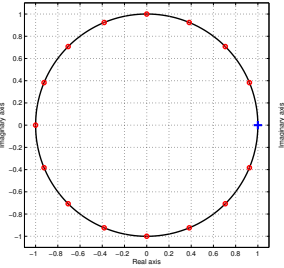

(b) $a=1$.

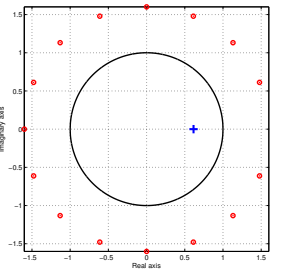

(c) $a=1.6$.

Fig. 9: Zero diagram for a $\ell_{1}$ filter and a antisparse signal. Another case where the filter zero remained inside the unit circle.

As we can see, the $\ell_{1}$ filter places a zero outside the unit circle for the Bernoulli-Gaussian signal (8c), which has a sparse structure to be explored. For this kind of signal, deconvolution was well succeeded even in maximum phase cases and the filter provides both magnitude and phase equalization.

However, in both cases of Figures 7 (Gaussian distribution) and 9 (uniform distribution), where there the input signal is not sparse, the filter zero remains inside the unit circle, showing the same limitation of the $\ell_{2}$ case.

Repeating the experiment (i.e., one coefficient for the filter, a $K=15$ order channel, optimization carried out by differential evolution with 40 individuals, in 2000 generations and signals with 2000 samples) for $\ell_{\infty}$ filter, we have the results in Figures 10, 11 and 12:

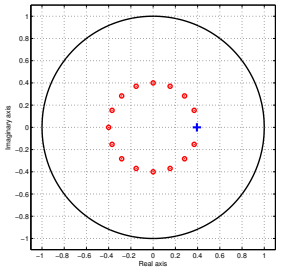

(a) $a=0.4$.

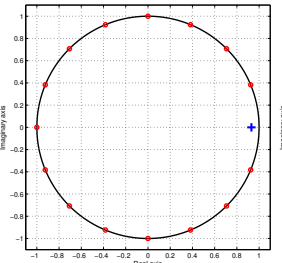

(b) $a=1$.

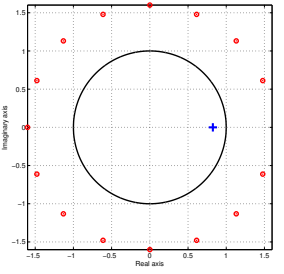

(c) $a=1.6$.
Fig. 10: Zero diagram for a $\ell_{\infty}$ filter and a Gaussian signal. As occurred with the $\ell_{1}$ case, the filter cannot put its zero outside the unit circle.

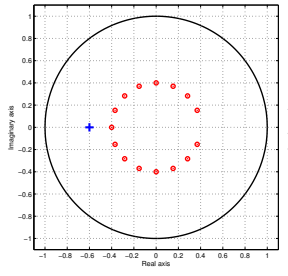

(a) $a=0.4$.

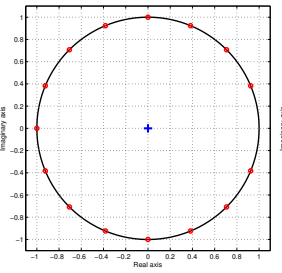

(b) $a=1$.

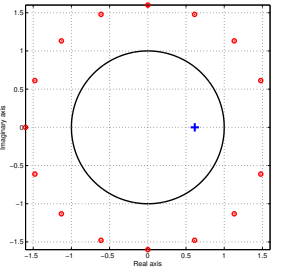

(c) $a=1.6$.
Fig. 11: Zero diagram for a $\ell_{\infty}$ filter and a Bernoulli-Gaussian signal. Again, the filter zero remained inside the unit circle, as a consequence of the signal distribution.

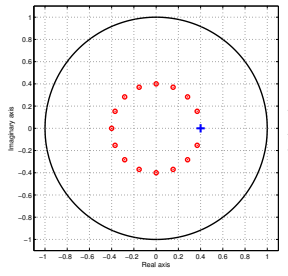

(a) $a=0.4$.

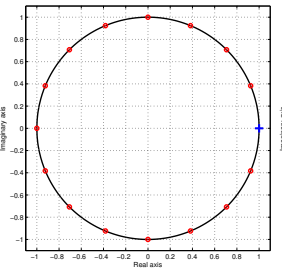

(b) $a=1$.

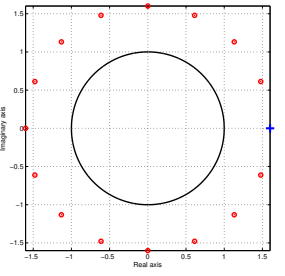

(c) $a=1.6$
Fig. 12: Zero diagram for a $\ell_{\infty}$ filter and a uniform signal. When we use an antisparse signal, the $\ell_{\infty}$ PEF put its zero outside the unit circle.

From these results, we can see that the $\ell_{\infty}$ filter places a zero outside the unit circle (Figure 12c) and performs magnitude and phase deconvolution for maximum phase channels. The key feature for this is that the input signal has a uniform (antisparse) distribution, which is the suitable one for the $\ell_{\infty}$ norm. For the other two distributions, no zero was positioned outside the unit circle, as seen in Figures 10 and 11, and only magnitude equalization is provided, as in the $\ell_{2}$ case.

Therefore, we can see that the $\ell_{p}$ PEFs have the potential to perform blind deconvolution in nonminimum phase channels, as long as the input signals have compatible structures (like sparsity or antisparsity). Exploring such characteristics, the $\ell_{p}$ norm can go further the decorrelation condition and towards to independence, as desired.

To close our study, in the next section we show some performance results in the deconvolution of first and second order channels, using $\ell_{1}$ and $\ell_{\infty}$ PEFs and comparing them with the classical $\ell_{2}$ PEF. 


\section{RESUlts ON THE PERFORMANCE OF THE $\ell_{p}$ PEF IN BLIND DECONVOLUTION}

Now let us evaluate the performance of the $\ell_{1}$ deconvolution for a sparse signal, and the $\ell_{\infty}$ deconvolution for an antisparse signal. Our results consider first and second order channels, of both minimum and maximum phases.

For the first order case, we have varied the channel (real) zero from 0.1 to 1.5 . For the second order channel we have used complex-conjugated zeros in polar coordinates:

$$
z_{1}=r e^{j \theta}, z_{2}=r e^{-j \theta} .
$$

In this case, we have varied $r$ in the interval $[0.1,1.5]$ and $\theta$ in $\left[\frac{\pi}{2}, \pi\right]$.

In all cases, we used a $L=10$ order filter, with coefficients adjusted by differential evolution; for the $\ell_{1}$ filter we have used a population of 20 individuals in 2000 generations and for the $\ell_{\infty}$ filter we used 30 individuals in 4000 generations.

To decide whether deconvolution was well succeeded, we considered the inter symbol interference (ISI), in decibel scale $(d B)$, of the global response (i.e. $g(n)=h(n) * w(n))$ :

$$
I S I_{d B}(g(n))=10 \log _{10} \frac{\sum_{i}\left|g_{i}^{2}\right|-\max _{i}\left|g_{i}\right|^{2}}{\max _{i}\left|g_{i}\right|^{2}} .
$$

When $I S I_{d B}(g(n)) \leq-5 d B$, the channel was considered equalized. For a better visualization, we will show our results in a zero diagram in complex plane: the red circles in the diagram represent the channel zero (or complex-conjugated zeros) for which deconvolution was well succeeded (accordingly to (27)). For example, if the channel with a zero at 0.2 was equalized, we will have a red circle at 0.2 in the complex plane.

Figure 13 presents the results for sparse deconvolution, using $\ell_{1}$ and $\ell_{2} \mathrm{PEF}$ :
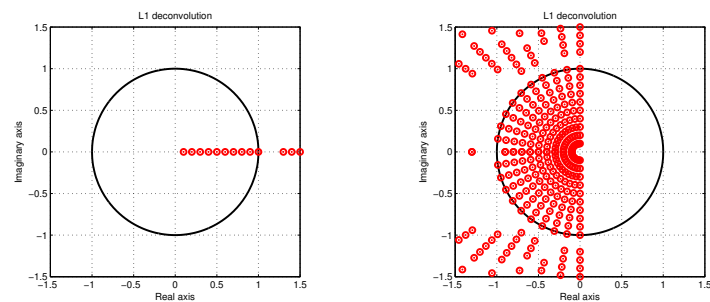

(a) First order channel norm.

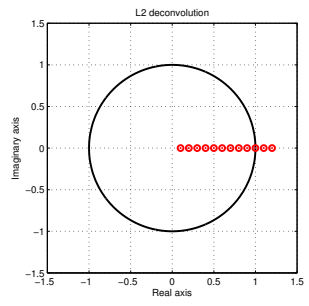

$\ell_{1}$ (b) Second order channel, $\ell_{1}$ norm.

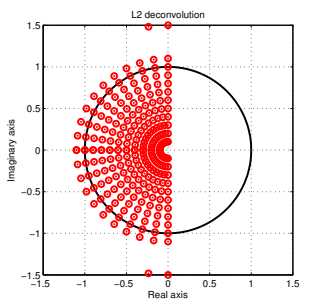

(c) First order channel, $\ell_{2}$ (d) Second order channel, $\ell_{2}$ norm. norm.

Fig. 13: Sparse deconvolution for first and second order channels, using $\ell_{1}$ and $\ell_{2}$ norm. The red circles represent the equalized channel zeros.
Figure 13 shows the zeros of the equalized channels, i.e., channels that achieve the threshold set for $I S I_{d B}(g(n))$, when using $\ell_{1}$ and $\ell_{2}$ PEF for the retrieval of a sparse signal. As Figures $13 \mathrm{a}$ and $13 \mathrm{~b}$ show, $\ell_{1}$ PEF performs deconvolution in maximum phase channels, but not in all of them (ideally, we must have all the left half of the complex plane marked, indicating a well succeeded deconvolution for all channels). This result shows both the potential and limitation of this criterion. On the other hand, the $\ell_{2}$ PEF performs deconvolution essentially in minimum phase channels (although we can see some maximum phase channels near the unit circle), as expected due to the restriction of the $\ell_{2}$ norm.

We obtained very similar results for the $\ell_{\infty}$ deconvolution, with antisparse signal, as shown in Figure 14:
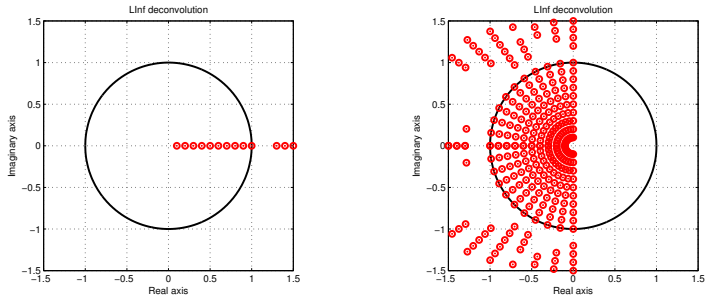

(a) First order channel, $\ell_{\infty}$ (b) Second order channel, $\ell_{\infty}$ norm.

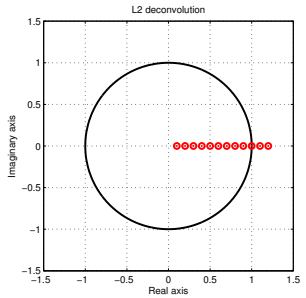
norm

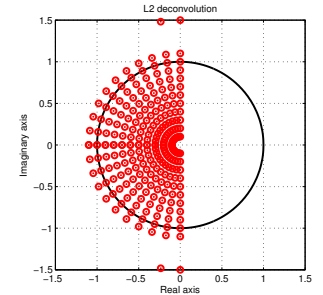

(c) First order channel, $\ell_{2}$ (d) Second order channel, $\ell_{2}$ norm. norm

Fig. 14: Antisparse deconvolution for first and second order channels, using $\ell_{\infty}$ and $\ell_{2}$ norm. The red circles represent the equalized channel zeros.

As illustrated by Figures $14 \mathrm{a}$ and $14 \mathrm{~b}$, the $\ell_{\infty}$ PEF also performs blind deconvolution in some nonminimum phase channels. For the $\ell_{2}$ filter with an antisparse signal we have the same limitations as before. Comparing Figures $13 \mathrm{a}$ and $13 \mathrm{~b}$ with $14 \mathrm{a}$ and $14 \mathrm{~b}$, respectively, we can see again the duality between the $\ell_{1}$ and $\ell_{\infty}$ filters, since the channels equalized in both cases are very similar.

Another interesting result is that there is a gap near the unit circle (radius 1.1) between the channels equalized by $\ell_{2}$ filters and those equalized by $\ell_{1} / \ell_{\infty}$ ones: at the point that the $\ell_{2}$ is no longer effective (i.e., from where the red circles are no longer present), we can apply the $\ell_{1} / \ell_{\infty}$, depending on signal structure, to carry out the deconvolution. This suggests that these criteria can be used together, in a cascaded hybrid-norm filter: the first filter could be adjusted by $\ell_{2}$ norm, dealing with the minimum phase component of the system, and the second one by $\ell_{1} / \ell_{\infty}$ norm, dealing with the maximum phase component left. 
Finally, we analyze the $I S I(g(n))$ evolution while the filter's coefficients are adjusted. For this, we considered two particular channels: a first order one, with a zero at 1.5 and a second order one, with $r=1.3$ and $\theta=7 \pi / 10$. For each channel, we have used a $\ell_{1}$ PEF for sparse deconvolution and a $\ell_{\infty} \mathrm{PEF}$ for the antisparse case. We show these results in Figure 15:

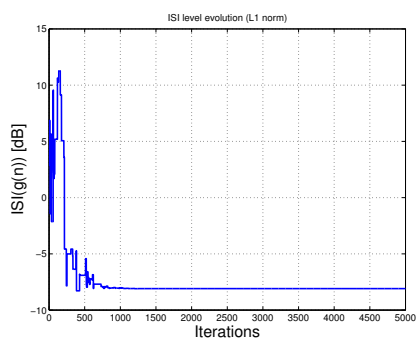

(a) First order channel, sparse deconvolution.

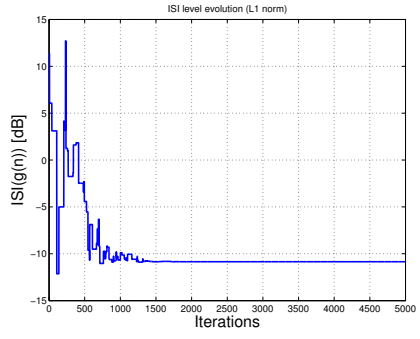

(c) Second order channel, sparse deconvolution.

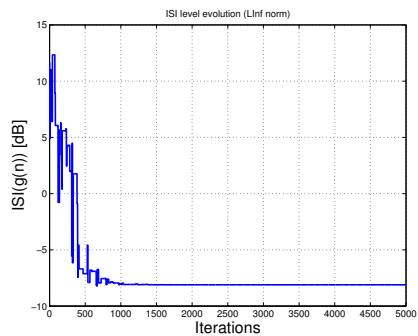

(b) First order channel, antisparse deconvolution.

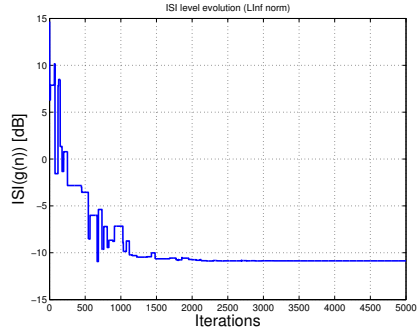

(d) Second order channel, antisparse deconvolution.

Fig. 15: $I S I(g(n))$ evolution during the filter adaptation, for first and second order channels, in sparse and antisparse deconvolution.

For the first order channel, we can see that the $\operatorname{ISI}(g(n))$ level reaches its steady value in 1500 iterations, for both sparse and antisparse cases. In the second order channel, we have a constant $I S I(g(n))$ level in 1500 iterations for the sparse scenario, and in 2000 iterations for the antisparse one.

As we can see in Figure 15, the Differential Evolution approach has a fast converge rate, and once the steady value is reached, we do not observe the typical misadjustments of a gradient approach.

\section{CONCLUSION}

This work discussed the application of $\ell_{p}$ norms in unsupervised deconvolution problem, using a predictive structure. We have shown that the $\ell_{p}$ criterion corresponds to the Maximum Likelihood estimator for generalized Gaussian distributions. Supported by this relation, we have applied a $\ell_{1}$ PEF in order to retrieve a sparse signal and a $\ell_{\infty}$ PEF for an antisparse one. Our experiments illustrated that both filters could place some of its zeros outside the unit circle, providing nonminimum phase responses.

We also evaluated $\ell_{1}$ and $\ell_{\infty}$ deconvolution, for first and second order channels, with minimum and maximum phase responses. We compared the results of these two experiments with the classical $\ell_{2}$ deconvolution. For a suitable input signal, i.e., a sparse signal for the $\ell_{1}$ case and an antisparse signal for the $\ell_{\infty}$, the proposed alternative criteria have shown a superior performance when compared to the classical $\ell_{2}$ deconvolution, especially for nonminimum phase distorting systems.

Above all, the present work confirms the potential of $\ell_{p}$ predictors in overcoming the minimum phase restriction, which open interesting perspectives in different applications of unsupervised deconvolution. In this sense, we are particularly interested in testing the proposed approach in more realistic models for telecommunications channels and for geophysics. As far as theoretical investigations are concerned, future works will focus on the application of the $\ell_{p}$ norms alongside with alternative filter structures, as well as in the extension to other challenging problems, like Blind Source Separation and multichannel equalization.

\section{APPENDIX}

\section{Description of DifFERENTIAL Evolution HeURistic}

In this appendix we describe the meta heuristic of differential evolution, which has been employed in the present work. The differential evolution meta heuristic uses a population of $P$ individuals, formed by $K$-dimensional vectors, at each generation $G$. In our application, each individual represents a prediction error filter with $K+1$ coefficients (i.e. a individual $\mathbf{w}_{\mathbf{i}, \mathbf{G}}=\left[\begin{array}{llll}w_{i 1} & w_{i 2} & \ldots & w_{i K}\end{array}\right]$ is associated with the filter $\left.\mathbf{w}\left(\mathbf{w}_{i, G}\right)=\left[1-w_{i 1}-w_{i 2} \ldots-w_{i K}\right]\right)$.

First, we randomly initialize the population in a way to better explore the search space. In cases where one has some information about the solution, like promising regions or even partial solutions, it can be used in the initialization process; otherwise, the population must be uniformly initialized, as done here: for each individual in the population, we generate $K$ independent and uniformly distributed random variables in the interval $[-1,1]$, for each candidate set of filter coefficients.

Differential evolution generates new individuals by a mutation process. This processes is performed by the addition of weighted differences between two vector to a third one, as shown next:

$$
\mathbf{t}_{i . G+1}=\mathbf{w}_{r_{1}, G}+F\left(\mathbf{w}_{r_{3}, G}-\mathbf{w}_{r_{2}, G}\right),
$$

where $\mathbf{t}_{i, G+1}$ is the new generated vector; $\mathbf{w}_{r_{l}, G}$, with $l=$ $1,2,3$, are individuals present in the population at generation $G$; and $r_{1}, r_{2}, r_{3}$ are mutually different indexes in the interval $[1, P]$ and also different from $i . F$ is a real constant in the range $[0,2]$, which determines the step towards the difference vector $\mathbf{w}_{r_{3}, G}-\mathbf{w}_{r_{2}, G}$.

Once the mutation is done, the mutated vectors are combined with the target vectors, generating the called trial vectors, accordingly with the following rule:

$$
z_{j i, G+1}= \begin{cases}t_{j i, G+1}, & \text { if } r_{j} \leq C R \text { or } j=l_{i}, \\ w_{j i, G}, & \text { if } r_{j}>C R \text { and } j \neq l_{i},\end{cases}
$$

where $\mathbf{z}_{i, G+1}=\left[\begin{array}{lllll}z_{1 i} & z_{2 i} & \ldots & z_{j i} & \ldots \\ z_{P i}\end{array}\right]$ is a trial vector, with $j=1,2, \ldots D ; r_{j}$ is a random variable uniformly distributed in the interval $[0,1] ; C R \in[0,1]$ its a real constant defined accordingly to the application and defines the crossover rate; and $l_{i}$ is a randomly chosen index in the interval $[1, P]$, 
which guarantees that $\mathbf{z}_{i, G+1}$ receives at least one component of $\mathbf{t}_{i, G+1}$.

Finally, we have the selection step. If the trial vector's fitness value is better than the fitness of the associated target value, the first takes the place of the latter in the next generation. Here, we have adopted the following fitness function:

$$
\operatorname{fitness}\left(\mathbf{w}_{\mathbf{i}, \mathbf{G}}\right)=\frac{1}{\|\mathbf{y}\|_{p}^{p}+1},
$$

where $\mathbf{y}=\left[\begin{array}{lll}y(0) & y(1) \ldots y(N-1)\end{array}\right]$ is the signal generated at the output of the filter associated with $\mathbf{w}_{i, G}$.

For the $\ell_{\infty}$ norm case, we have used the min max estimator, so the fitness function is given by:

$$
\operatorname{fitness}\left(\mathbf{w}_{\mathbf{i}, \mathbf{G}}\right)=\frac{1}{\max (|\mathbf{y}|)+1} \text {. }
$$

All the process described above (mutation, crossover and selection) are repeated until the maximum number of generations is achieved. Once the process is concluded, we take the best individual (i.e. the one with the highest fitness value) as the solution to our problem.

\section{REFERENCES}

[1] A. N. Kolmogorov, "Sur l'interpolation et l'extrapolation de suites stationaires." Comtes Rendus de l'Académic de Sciences, vol. 207, pp. 2043-2045, 1939

[2] N. Wiener, Extrapolation, interpolation, and smoothing of stationary time series. New York: Wiley, 1949.

[3] J. M. T. Romano, R. Attux, C. C. Cavalcante, and R. Suyama, Unsupervised signal processing: channel equalization and source separation. CRC Press, 2011.

[4] K. Nose-Filho, "Adaptive sparse linear prediction: a promising tool for blind deconvolution," IEEE Sensor Array and Multichannel Signal Processing Workshop (SAM), 2016, DOI:10.1109/SAM.2016.7569713.

[5] P. P. Vaidyanathan, The theory of linear prediction. Morgan \& Claypool Publishers, 2008.

[6] M. Najim, Modeling, Estimation and Optimal Filtering in Signal Processing. John Wiley and Sons, Inc., 2008.

[7] G. E. P. Box, G. M. Jenkins, and G. C. Reinsel, Time Series Analysis: Forecasting and Control. Wiley, 2008.

[8] O. Macchi, C. A. Faria, and J. M. T. Romano, "Egalisation adaptive autodidacte par rétroprédiction et prédiction," in Proceddings of the Colloque GRETSI, 1993.

[9] J.-M. Brossier, Signal et communication numérique: égalisation et synchronisation. Hermes, 1997.

[10] R. D. B. Brotto, K. Nose-Filho, and J. M. T. Romano, "Critérios alternativos para desconvolução não supervisionada," $8^{\circ}$ Simpósio de Instrumentação e Imagens Médicas/ $7^{\circ}$ Simpósio de Processamento de Sinais, 2017.

[11] C. Elvira, P. Chainais, and N. Dobigeon, "Bayesian antisparse coding," IEEE Transaction on Signal Processing, vol. 65, pp. 1660-1672, 2015, DOI:https://doi.org/https://doi.org/10.1109/TSP.2016.2645543.

[12] E. A. Robinson, "Predictive decomposition of time series with applications to seismic exploration," Ph.D. dissertation, Massachusetts Institute of Technology, 1954.

[13] A. V. Oppenhein, A. S. Willsky, and S. H. Nawab, Signals and systems. Prentice Hall, 1996.

[14] K. Nose-Filho, "Desconvolução e separação cega de sinais esparsos e aplicações em sísmica de reflexão," Ph.D. dissertation, Faculdade de Engenharia Elétrica e de Computação da Universidade Estadual de Campinas, 2015.

[15] J. Proakis and M. Salehi, Digital Communications. McGraw-Hill Education, 5th ed., 2007.

[16] A. Benveniste, M. Goursat, and G. Ruget, "Robust identification of a nonminimum phase system: blind adjustment of a linear equalizer in data communications," IEEE Transactions on Automatic Control, vol. 25, no. 3, pp. 385-399, 1980, DOI:10.1109/TAC.1980.1102343.
[17] O. Shalvi and E. Weinstein, "New criteria for blind deconvolution of nonminimun phase systems (channels)," IEEE Transactions on Information Theory, vol. 36, pp. 312-321, 1990, DOI:10.1109/18.52478.

[18] A. Papoulis and S. U. Pillai, Probability, Random Variables and Stochastic Processes. McGraw-Hill Europe, 2002.

[19] J. R. Rice and J. S. White, "Norms for smoothing and estimation," SIAM Review, vol. 6, no. 3, pp. pp. 243-256, 1964. [Online]. Available: http://www.jstor.org/stable/2027590

[20] A. Bell and T. Sejnowski, "An information-maximization approach to blind separation and blind deconvolution," Neural Computation, vol. 7, pp. 1129-1159, 1995, DOI:https://doi.org/10.1162/neco.1995.7.6.1129.

[21] P. Comon and C. Jutten, Eds., Handbook of blind source seapartion. Academic Press, 2010.

[22] J.-F. Cardoso, "Blind signal separation: Statistical principles," Proceedings of IEEE, vol. 86, pp. 2009-2025, 1998, DOI:https://doi.org/10.1109/ 5.720250 .

[23] S. M. Kay, Fundamentals of Statistical Signal Processing: Estimation Theory. Prentice Hall, 1993.

[24] S. Nadarajah, "A generalized normal distribution," Journal of Applied Statistics, vol. 32, n.7, pp. 685-694, 2005, DOI:https://doi.org/10.1080/ 02664760500079464.

[25] J. Makhoul, "Linear prediction: a tutorial review," Proceedings of the IEEE, vol. 63, pp. 561-580, 1975, DOI:https://doi.org/10.1109/PROC 1975.9792.

[26] G. Cybenko, "Locations of zeros of predictor polynomials," IEEE Transactions on Automatic Control, vol. Ac-27, pp. 235-237, 1982, DOI:https://doi.org/10.1109/TAC.1982.1102870.

[27] J. M. T. Romano and M. Bellanger, "Zeros and poles of linear prediction digital filter," in Proceedings of the 1986 EUSIPCO, 1986.

[28] J. D. Scargle, "Absolute value optimization to estimate phase properties of stochastic time series," IEEE Transactions on Information Theory, vol. 23, no. 1, pp. 140-143, 1977, DOI:https://doi.org/10.1109/TIT.1977. 1055668.

[29] T. W. J. Bednar, R. Yarlagadda, " $\ell_{1}$ deconvolution and its application to seismic signal processing," IEEE Transactions on Acoustics, Speech and Signal Processing, vol. 34, no. 6, pp. 1655-1658, 1986, DOI:https: //doi.org/10.1109/TASSP.1986.1165004.

[30] L. Knockaert, "Stability of linear predictors and numerical range shift operators in normal spaces," IEEE Transactions on Information Theory, vol. 38, no. 5, pp. 1483-1486, 1992, DOI:https://doi.org/10.1109/18. 149499.

[31] S. Haykin, Adaptive filter theory. Prentice Hall, 2001.

[32] J. A. L. K. Price, R. M. Storn, Differential Evolution: A Practical Approach to Global Optimization. Natural Computing Series, Springer, 2005.

[33] J. W. Brown and R. V. Churchill, Complex variables and applications. McGraw-Hill, 2009.

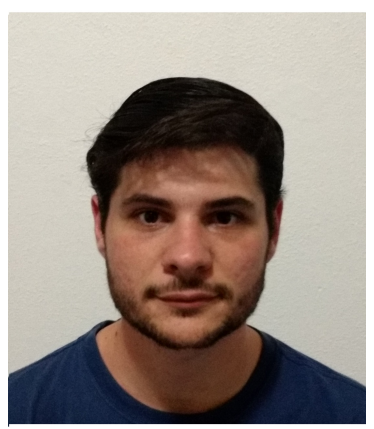

Renan Del Buono Brotto was born in Serra Negra, Brazil, in 1993. $\mathrm{He}$ obtained the B.S. degree in Computer Engineering from State University of Campinas, Brazil, in 2016. He is currently pursuing the Master degree at the same university in the Digital Signal Processing for Communications Laboratory. His research interests are unsupervised signal processing, artificial intelligence and applications in communications and geophysics. 


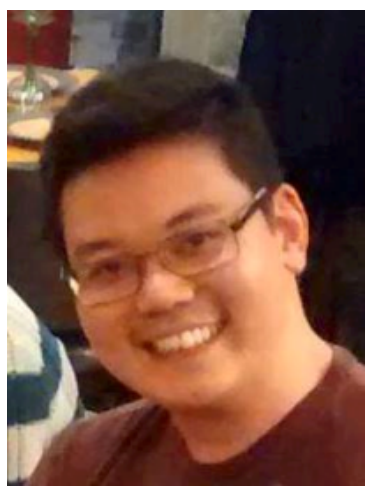

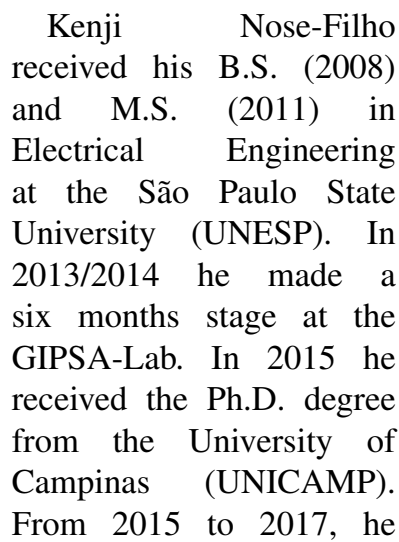

made a post-doc at the University of Campinas (UNICAMP). His main research interest is in digital signal processing and machine learning, with applications in geophysics, time series forecasting, and image processing. He has been an assistant professor at the Engineering, Modeling and Applied Social Sciences Center at the Federal University of ABC since 2017.

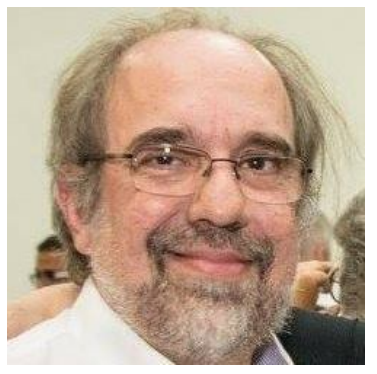

João Marcos Travassos was born in Rio de Janeiro on April 12, 1960. He obtained the BSc and MSc degrees in Electrical Engineering at the University of Campinas (UNICAMP), in 1981 and 1984 respectively. He received his $\mathrm{PhD}$ from the University of Paris-XI in 1987.

Since 1988, he is at the School of Electrical and Computer Engineering (FEEC) of UNICAMP, where he is currently Professor. He was Visiting Professor at the CNAM/Paris and the Université René Descartes - Paris.

Prof. Romano has been Head of the Communications Department of FEEC/UNICAMP, Associate Coordinator of Graduate Program in Electrical Engineering, Associate Director of FEEC, and Director of Partnerships and Collaborative Projects of the Innovation Agency of the university (INOVAUNICAMP). Currently, he is the director of the School of Electrical and Computer Engineering at UNICAMP.

Prof. Romano coordinates the research activities of the Signal Processing for Communications (DSPCom) Lab, where he works in the areas of adaptive filtering, unsupervised learning, blind source separation and signal processing applications in communications and geophysics. He has a $\mathrm{CNPq}$ research grant, level 1B.

Prof. Romano has been Vice-President (1996-2000) and President (2000-2004) of the Brazilian Telecommunications Society (SBrT), and is an Emeritus Member of SBrT. He has been the Editor-in-Chief of the SBrT/IEEE JCIS from 2011 to 2014 and he is currently a member of SBrT's Deliberative Council. 